\title{
TORQUE MAXIMISATION OF THE PMAC MOTOR FOR HIGH PERFORMANCE, LOW INERTIA OPERATION
}

\author{
Paul Stewart
}

\begin{abstract}
This paper describes the techniques applied to maximise the torque envelope of the permanent magnet AC (PMAC) motor operating under current and voltage constraints. Standard steady-state descriptions of the system are often suitable for control purposes when the rotor velocity is varying relatively slowly. In low inertia applications such as clutchless gearchange operations, where in the pursuit of driveability, the motor is required to accelerate and decelerate its own rotor inertia as quickly as possible. In this case, the voltage drop due to the current dynamics start to become significant. This paper presents a method to reserve voltage headroom dynamically in the field-weakening region in order to maximise the torque envelope when the effective inertia is low. Experimental results show the effectiveness of this approach.
\end{abstract}

KeyWords: Permanent magnet AC synchronous motor, model reference, field weakening, torque control.

\section{INTRODUCTION}

Torque maximisation for smooth rotor (surface mounted magnets) permanent magnet machines has been addressed [1] using steady-state (i.e. constant rotor velocity) system description with the aim of yielding smaller motors for given applications, or faster operation for a given size of motor. The major control problem when considering both steady state and dynamic operation of the PMAC motor can be seen to be the finite voltage and current supply available from the DC link and the inverter. In order to maximise the torque envelope, it is necessary to apply field-weakening (advancing the phase of the current vector to suppress the motor EMF) techniques which are derived assuming that rotor velocity is constant. The assumption is made that the rotor velocity varies slowly enough so that the steady state assumptions are valid [2]. The problem has also been addressed in [3], however since the approach is geometrical, stator resistance is neglected. The PMAC motor will be analysed in the d-q synchronous reference frame which is obtained via a non linear transform. It

Manuscript received March 5, 2001; revised December 18, 2001; accepted January 7, 2002.

P. Stewart is with the Department of Electronic and Electrical Engineering, University of Sheffield, Mappin Street, Sheffield S1 3JD, United Kingdom. will be shown that as the machine's rotor accelerates, the rising value of back EMF restricts the magnitude of torque producing current until further torque production is not possible. Field-weakening control [10] will be implemented in such a manner which makes possible a controller which effectively increases the useful speed range of the motor, and also takes account of the voltage drop due to the current dynamics and phase resistance when operating as a low inertia, high performance drive.

\section{THE CONSTRAINED SYSTEM DESCRIPTION}

In the d-q model of the PMAC motor, the stator currents are transformed into an orthogonal frame of reference which is moving synchronously with the rotor flux. The orthogonal reference frame is derived from the phase currents via the nonlinear transform [5-7]

$\left[\begin{array}{l}V_{q} \\ V_{d}\end{array}\right]=\frac{2}{3}\left[\begin{array}{ccc}\cos (\theta) & \cos \left(\theta-\frac{2 \pi}{3}\right) & \cos \left(\theta+\frac{2 \pi}{3}\right) \\ \sin (\theta) & \sin \left(\theta-\frac{2 \pi}{3}\right) & \sin \left(\theta+\frac{2 \pi}{3}\right)\end{array}\right]\left[\begin{array}{c}V_{a} \\ V_{b} \\ V_{c}\end{array}\right]$

where $V_{d}, V_{q}$ are the $\mathrm{d}$ and q axis voltages, $V_{a, b, c}$ are the three phase elements and $i_{d}, i_{q}$ are the $d$ and $q$ axis currents. Which gives the system voltage drops in the synchronous frame 


$$
\begin{aligned}
& V_{q}=r i_{q}+\omega L i_{d}+\omega k+L\left(\frac{d i_{q}}{d t}\right) \\
& V_{d}=r i_{d}-\omega L i_{q}+L\left(\frac{d i_{d}}{d t}\right)
\end{aligned}
$$

Where $r$ is the phase resistance, $\omega$ is the rotor velocity, $L$ the phase inductance and $k$ the back EMF constant in the reference frame as volts/radians/second. Electrical torque developed by the motor is proportional to the q-axis current $T_{e}=k_{t} i_{q}$ where $k_{t}$ is the motor torque constant. This transformation also applies to current and flux linkage quantities, and the three phase quantities may be obtained from the $d$ and $q$ axis variables by application of an inversion of the matrix in equation (1). The q-axis inductance is equivalent to the armature inductance, and the $\mathrm{d}$-axis inductance is equivalent to the field inductance. In the case of the surface mount PMAC motor, these quantities are equal. High speed operation of the PMAC machine is constrained by the linear proportionality of the back-EMF and inductive voltage drops to rotor velocity. The system operates subject to the constraints (4) on voltage and current,

$$
V_{s}^{2} \geq V_{q}^{2}+V_{d}^{2} \quad I^{2} \geq i_{q}{ }^{2}+i_{d}{ }^{2}
$$

where $V_{s}$ is the magnitude of the voltage vector, and $I$ is the magnitude of the current vector. The amount of current that can be supplied by the three phase inverter is limited by the heat dissipation properties of the motor, and by the current rating of the inverter. It is also limited by the converter DC link voltage, which must overcome both the EMF, and the voltage drops across the synchronous reactance and resistance. Considering the system constraints, maximum torque-per-amp operation below base speed requires the $d$-axis current to be controlled to zero. Above this boundary, the rising EMF value can be countered by advancing the current vector with respect to the rotor (field or flux weakening), and introducing negative $d$-axis current The requirements of the flux weakening controller are to calculate the velocity at which flux-weakening is to be initiated, and to output optimal $d$-axis and $q$-axis current commands in steadystate and dynamic operation.

\section{TORQUE MAXIMISATION}

If for the moment, resistance is neglected, then from equations (2) and (3) the steady-state system becomes equations (5), (6), and the instantaneous torque $T_{e}$ for the surface mount PMAC motor is given [4] by equation (7), where $k_{t}$ is the motor torque constant.

$$
V_{q}=\omega L i_{d}+\omega k
$$

$$
\begin{aligned}
& V_{d}=-\omega L i_{q} \\
& T_{e}=k_{t} i_{q}
\end{aligned}
$$

The current limit is defined by a circular locus defined by equation (4). Similarly, the DC supply voltage limits the voltage vector to a circular locus with frequency dependant radius, and different center $\left(\frac{-k}{L}, 0\right)$. The voltage limit locus is derived from the identity in equation (4), then in steady-state:

$$
V^{2}=\omega^{2} L^{2} i_{q}{ }^{2}+\left(\omega k+\omega L i_{d}\right)^{2}
$$

and;

$$
\frac{V}{\omega L}=\sqrt{i_{q}^{2}+\left(i_{d}+\frac{k}{L}\right)^{2}}
$$

As speed and frequency increase, the current limit locus remains fixed, however the radius of the voltage limit locus decreases. Eventually the PWM control saturates when its duty cycle reaches maximum, and the available sinewave voltage from the inverter equals the phase voltage. This operating point is known as 'base speed' and occurs at the intersection of the $q$ axis, current limit circle, and voltage limit circle. If the rotor velocity increases further, the radius of the voltage-limit circle decreases further, and maximum current is defined by a current vector terminating in the intersection of the two circles.

\subsection{Model reference control}

The operating principle of the model reference control systems is to generate optimal $d$ and $q$-axis command values to be tracked by PI controllers [8]. In this particular case, to maximise the torque speed envelope of the motor in the presence of voltage and current constraints. The model reference controller operates in a number of modes according to a decision tree (Fig. 1), and utilises the system equations to calculate the appropriate command values. Rearranging the system equation (2) gives the reference model

$$
i_{q}^{*}=V_{q}^{*}-\omega L i_{d}^{*}-\omega k
$$

and by substituting equations (10) and (3) into the supply voltage constrained voltage vector

$$
V=\sqrt{V_{q}^{* 2}+V_{d}^{* 2}}
$$

and the expression for instantaneous torque

$$
T_{e}^{*}=\frac{3 P}{2} \phi_{f} i_{q}^{*}=k_{t} i_{q}^{*}
$$




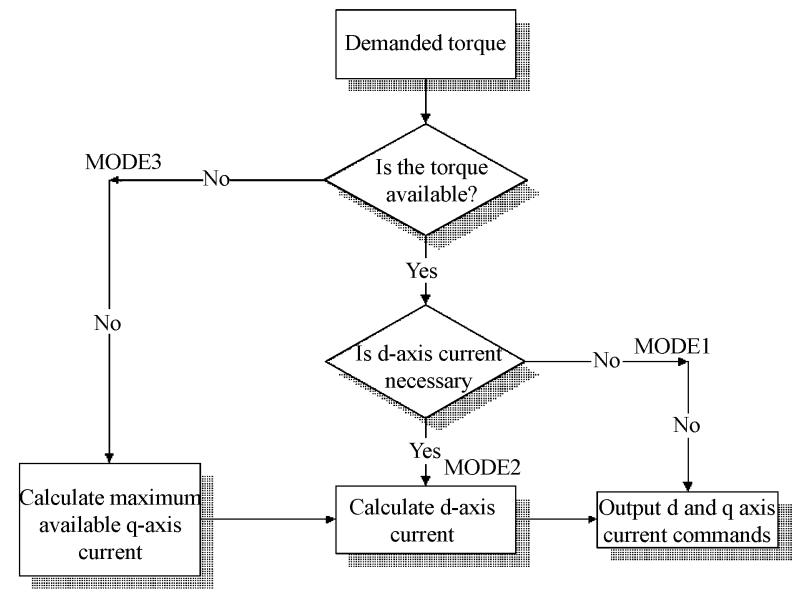

Fig. 1. Model reference controller decision tree.

yields an expression which describes the torque producing component of the system in terms of the physical parameters, torque constant $k_{t}$ and speed variable $\omega$;

$$
T_{e}^{*}=\frac{k_{t}}{\omega L} \sqrt{V^{2}-\omega^{2} k^{2}}=k_{t} \sqrt{\left(\frac{V}{\omega L}\right)^{2}-\left(\frac{k}{L}\right)^{2}}
$$

also

$$
V^{2}=\left(\omega L i_{d}^{*}+\omega k\right)^{2}-\left(\omega L i_{q}^{*}\right)^{2}
$$

Combining equations (13), (14) yields

$$
\left(\frac{V}{\omega L}\right)^{2}=\left(i_{d}^{* 2}+\frac{k}{L}\right)^{2}+\left(\frac{T_{e}^{*}}{k_{t}}\right)^{2}
$$

which describes the frequency dependent voltage vector in terms of the current components, and forms the model for calculating the system command outputs for the PMAC drive operating modes. The omission of the current dynamics has the fundamental consequence that the components which represent the advance of the current vector with respect to time are unmodelled, and consequently a maximum voltage drop of magnitude

$$
\Delta V=\sqrt{\left(L \frac{d i_{d}}{d t}\right)^{2}+\left(L \frac{d i_{q}}{d t}\right)^{2}}
$$

has been ignored. It is important to note here that in applications where the motor is driving significant loads, then the field-weakening controller based on the steadystate equations is perfectly satisfactory. However in the case of torque maximising field-weakening control with the rotor inertia only, then the current dynamics become significant. Thus when the PMAC enters the field weakening region and the current regulators saturate, no spare voltage is available to acommodate the dynamic

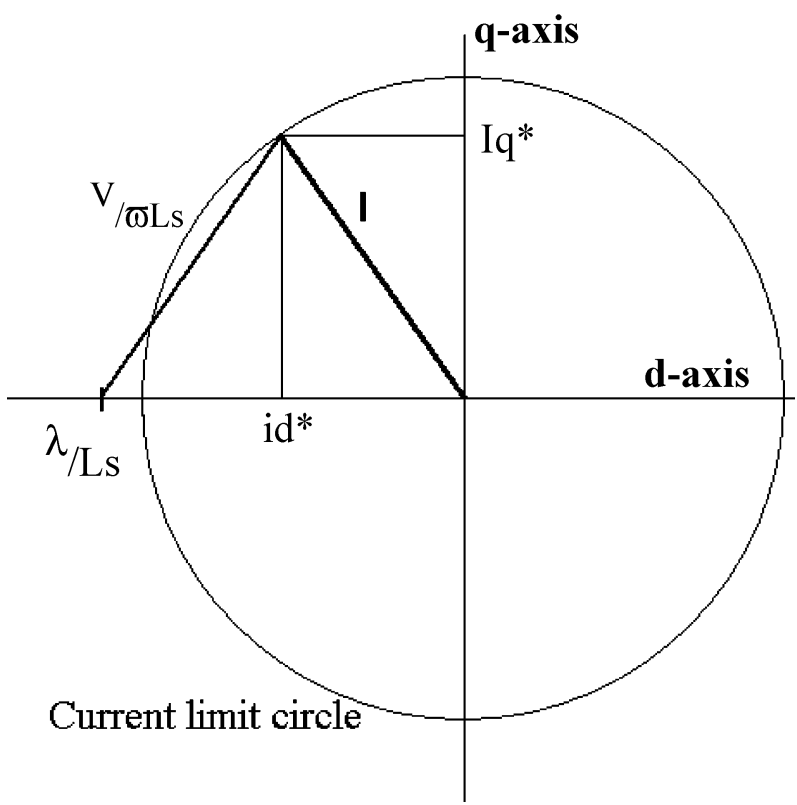

Fig. 2. PMAC motor as described by the steady state model reference implementation.

movement of the current vector, and consequently the optimal path for the maximum torque profile cannot be followed.

\subsection{Calculating the dynamic voltage drop}

An equation can be derived to describe the rate of change of the angle of the current vector in the $d-q$ reference frame as the rotor accelerates. Ignoring resistive effects, we obtain an expression in the steady state which relates the supply voltage to the rotor velocity.

$$
V^{2}=\omega^{2} L^{2} i_{q}^{2}+\left(\omega k+\omega L i_{d}\right)^{2}
$$

The control extension will be designed to reserve sufficient voltage headroom by dynamic allocation in the field weakening region, avoiding the torque-speed envelope degradation experienced with the steady state model reference controller. This expression in equation 17 is differentiated and solved for $\frac{d \sigma}{d t}$ where $\sigma$ is the angle of current vector advance ahead of the q-axis. In order to obtain a description of the current vector dynamics, using

$$
\frac{d \omega}{d t}=\frac{T}{J}=\frac{k_{t} I \cos \sigma}{J}
$$

we obtain an expression for the current vector advance angle which allows calculation from the system parameters and measured current, assuming that the limits $I$ and $V$ are treated as constants; 


$$
\frac{d \sigma}{d t}=\frac{-k_{t}\left(L^{2} I^{2}+2 L I \sin (\sigma) k+k^{2}\right.}{\omega k L J}
$$

which shows the dependancy on the system inertia. The derivatives of the $d-q$ currents are given by

$$
\begin{aligned}
& \left(\frac{d i_{q}}{d t}\right)=-i_{d}\left(\frac{d \sigma}{d t}\right) \\
& \left(\frac{d i_{d}}{d t}\right)=i_{q}\left(\frac{d \sigma}{d t}\right)
\end{aligned}
$$

and we obtain the voltage drop due to the current vector angle dynamics

$$
\Delta V=\frac{-k_{t} L I\left(L^{2} I^{2}+2 L_{s} I \sin (\sigma) k+k^{2}\right)}{\omega k L_{s} J}
$$

where $\Delta V$ is the dynamic voltage drop.

\subsection{Dynamic base speed}

Under dynamic conditions, the onset of field weakening will occur at a lower frequency than in the steady state due to the voltage necessary to drive the current vector phase dynamics. At this new virtual base speed, which represents the boundary between the constant torque and constant power regions, the $d$-axis current is set to zero, and the equation for dynamic voltage drop reduces to

$$
\Delta V=\frac{I L k_{t}\left(L^{2} I^{2}+k^{2}\right)}{\omega k J}
$$

An expression can be derived from the circle diagram description to link the difference between the magnitudes of the voltage-frequency limi circles at dynamic and steady state base speeds, where $V_{s}$ is the magnitude of the applied voltage.

$$
\Delta V=V_{s}-\sqrt{I^{2}+\omega L \frac{k^{2}}{L^{2}}}
$$

combining equations $(1.11,1.12)$ gives a quadratic in rotor velocity

$$
0=-\omega^{2} L \sqrt{I^{2}+\frac{k^{2}}{L^{2}}}+V_{s} \omega-\frac{I L k_{t}\left(L^{2} I^{2}+k^{2}\right)}{k J}
$$

The solution of this equation gives the difference between the dynamic and steady state base speed, and the dynamic base speed $\omega_{d}$ where,

$$
\omega_{d}=\omega_{o}-\Delta \omega
$$

and,

$$
\Delta \omega=\omega_{o}\left(\frac{V_{s} k_{t} I L_{s}}{k J \omega_{s}^{2}}\right)
$$

\subsection{Implementing the scheme\}}

In the steady state model reference controller, the current vector $I$ is calculated as extending from $(0,0)$ in the $d-q$ frame to the intersection of the current limit circle and the frequency related voltage limit circle with radius $\frac{V}{\omega L}$ and center $\left(-\frac{k}{L}, 0\right)$. The outputs of the reference model are the command quantities $i_{q}^{*}$ and $i_{d}^{*}$ (which are changing as the motor accelerates). In order to reserve sufficient voltage headroom to allow the current dynamics to act effectively, the length of the voltage limit vector $\frac{V}{\omega L}$ must be reduced by an appropriate amount. The voltage reserved for this effect has $d$-axis and $q$-axis components and can be described as being a vector extending from the voltage limit vector in the reference model. The $d$-axis and $q$-axis current commands are now formulated as the intersection of the dynamic voltage drop (which augments the steady state voltage limit vector) and the current limit circle. For the benefit of reduced computation in the model reference controller, the voltage drop due to the dynamics can be approximated as a worst case. That is the magnitude of the dynamic voltage drop is calculated and subtracted from the magnitude of the voltage limit vector, the new voltage limit vector including the dynamic components being represented as $V_{l}$.

$$
V_{l}=V_{s}-\Delta V
$$

Similarly, the unmodelled voltage drop due to resistive effects can be included as a worst case effect, and thus the voltage limit vector now contains all the significant system voltage drops, with any minor unmodelled drops being lumped together in the worst case adoption of vector directions. The voltage limit vector now becomes

$$
V_{l}=\frac{k}{\omega L}-\Delta V-r I
$$

If this voltage can be reserved in operation, coupled with a revised base speed to enable the reservation process, then the machine should follow the optimal current trajectory with current regulators held just within the bounds of saturation. The geometric implementation in the model reference controller is shown in Fig. 3.

\section{RESULTS}

Simulation of the model reference controller operating with an $87 \mathrm{~kW}$ surface mount traction motor indi- 


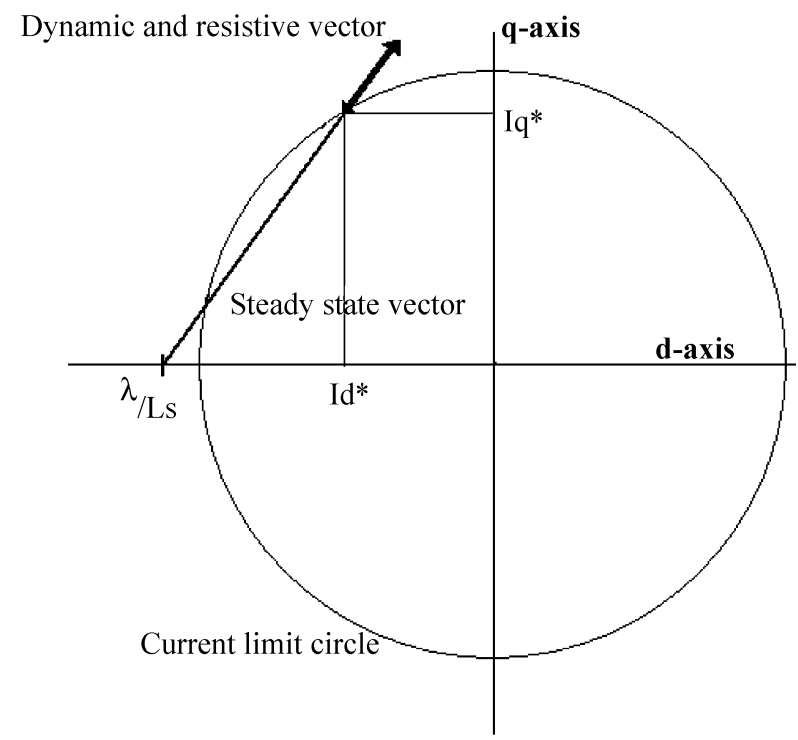

Fig. 3. PMAC dynamic model reference control geometric implementation.

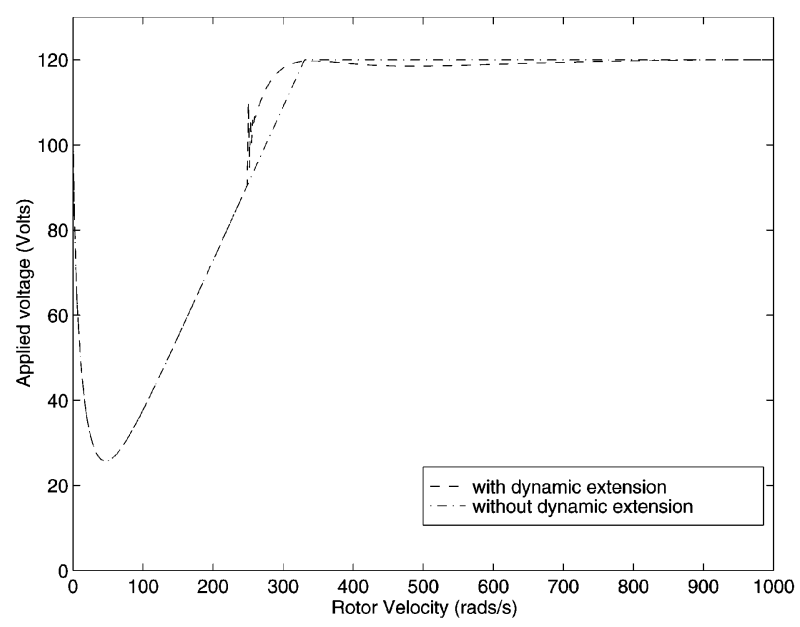

Fig. 4. PMAC simulated applied voltage magnitude.

cated the potential for experimental implementation of the torque maximising scheme. Firstly, of interest is the predicted voltage magnitude comparison shown in Fig. 4 The controller with the dynamic extension is seen to operate at a lower rotor velocity than the controller based on the steady-state description. Another interesting feature is that the controller does not saturate in the field weakening region, but operates just within saturation, thus retaining full control of the current vector. A velocity profile similar to that in a clutchless gearchanging operation was simulated in Fig. 5. The controller with the dynamic extension was found to have an advantage of approximately $50 \mathrm{~ms}$ at the end of the operation, an amount which in terms of high performance vehicle driveability, is significant. The model reference controller with the dynamic extension reserving voltage head

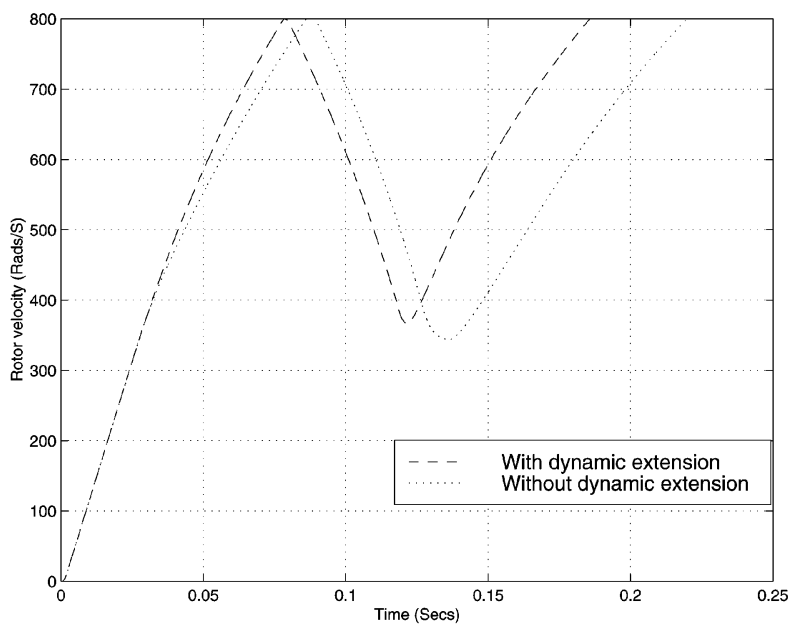

Fig. 5. Simulated velocity profile comparison.

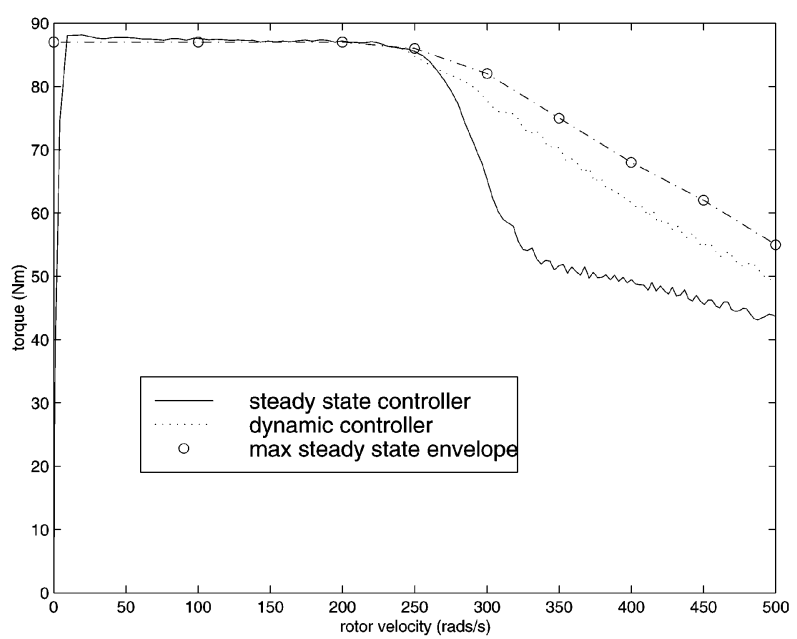

Fig. 6. Performance comparisons of the reference model controller with and without the dynamic extension. Rotor only inertial load.

room were implemented on a TI TMS320C5 digital signal processing board, controlling a PMAC motor on a four quadrant PWM controlled [11,12] dynamometer. Due to the dynamic bandwidth limitations of torque transducers, the instantaneous torque is calculated from the $q$-axis current. The first experiment is for the motor with just the rotor inertia and no external load. This is the most severe test due to rotor acceleration, and should show the greatest deterioration in torque output. The rotor is allowed to accelerate freely from rest under maximum torque demand in order to compare with the maximum torque envelope. The relative performance can be seen in Fig. 6. The optimal torque speed envelope is obtained by operating the motor at steady-state points, and ascertaining the maximum torque output on a dynamometer. This is labeled on the graph as max steady state envelope. This is a set of discrete torque measure- 


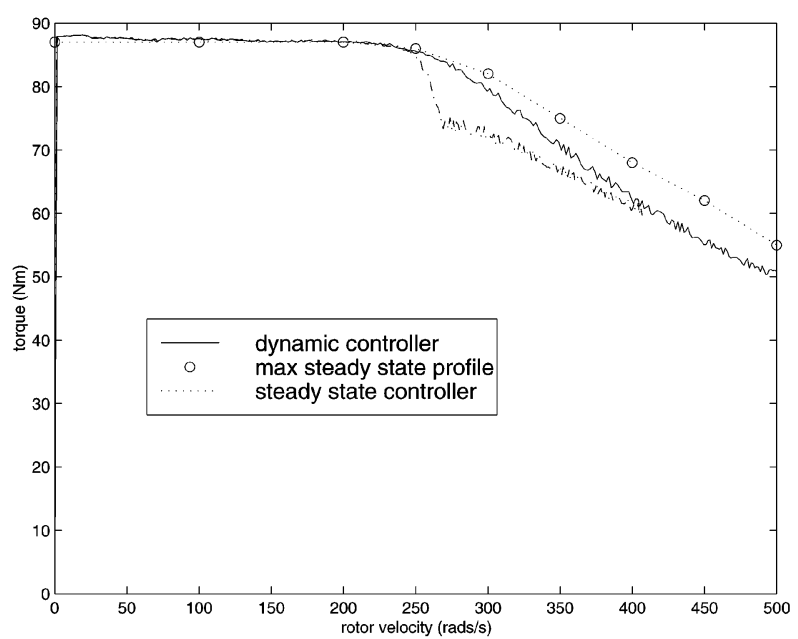

Fig. 7. Performance comparisons of the model reference controllerwith and without the dynamic extension. Rotor and external load motor inertial load.

ments taken at a range of speeds when all transients have decayed to zero, and represents the maximum torque which can be produced by the system at any speed. The series labelled steady state controller represents the maximum torque envelope produced by a machine operating dynamically with model reference being produced by the steady state representation. Finally, the series labelled dynamic controller is the maximum torque envelope of the motor freely accelerating, and being controlled by the model reference augmented by the dynamic voltage reservation scheme. The experiment shows that the dynamic controller has produced a greatly enhanced torque envelope when compared to the controller based on the steady-state system description. The difference in maximum torque at any point between the dynamic controller and the max steady state envelope is due to the fact that under steady state conditions, no voltage is required for the current vector dynamics, and consequently more voltage is available for torque production. The experiment of the motor driving an external load motor giving a substantially greater inertia was repeated with the dynamic controller. The comparison of the maximum torque envelope of this simulation is shown in Fig. 7. This maximum torque envelope is compared with the steady state controller operating dynamically with the same inertia and also the maximum steady state torque profile. The divergence between the performance of the dynamic and steady state model reference controllers is now markedly less pronounced as predicted. The torque profiles of the two regimes converge around $400 \mathrm{rads}^{-1}$ as the unmodelled dynamic voltage drop experienced by the steady state controller begins to diminish. The experiments confirm the requirement of extending the model reference controller with the dynamic voltage reservation scheme. The im-

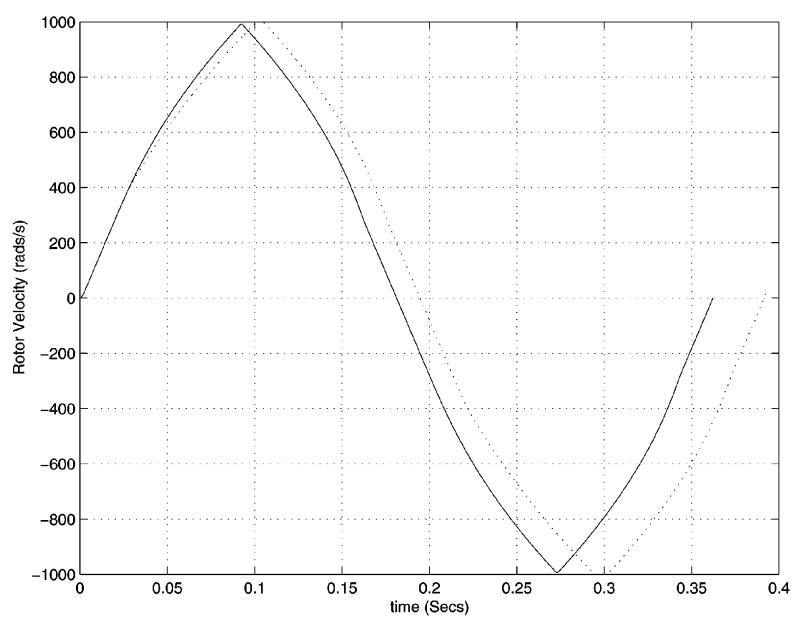

Fig. 8. Test velocity profile, comparison of dynamic and steady state based controller.

provement in performance when the motor is diconnected from its load is particularly pronounced. In the case of the traction application under study here, much of the dynamic operation takes place around base speed, and the enhanced torque profile made available by the dynamic scheme allows the fastest possible gearchanges. This enhancement would also be important for low inertia servo applications.

\section{CONCLUSION}

A controller has been designed, simulated and experimentally investigated, which maximises the torque speed envelope of the PMAC motor in low inertia operating mode. It has the advantages of relatively simple implementation, and in practice requires no tuning for any arbitary motor. The approach is found to have particular benefits for maximizing the torque envelope with low inertia applications, where the effects of the dynamic voltage drop are the most pronounced, due to the fast acceleration of the rotor. As shown in Fig. 8, the controller with the dynamic extensions is able to achieve faster transition times due to the elevated torque envelope which has been made available. This has been found to be of particular benefit in clutchless gearchanging on electric vehicles, where the concept of "driveability" indicates that lags which are only of the order of milliseconds can influence the driver's perception of the vehicle's performance. The application of the dynamic extension can contribute to the increase in perception of driveability, and is thus a useful implementation. The extension can be used with either the direct calculation, or current error methods of field weakening implementation, and has been found to be reasonable to implement in a real-time controller. Although the entire technique relies on motor parameters to effect the calculations, on-line parameter estimation could easily be added to the 
system to increase the controller's robustness [9] The geometrical approach to the model reference controller was found to be easy to implement on DSP, and has the advantage not only of including the current dynamics, but also including resistive voltage drops, which have been neglected in previous implementations of geometric controllers. A further refinement was added to the controller at a later stage, that is the minimisation of the magnitude of the current vector at all times in order to reduce $i^{2} r$ losses in the motor windings.

\section{APPENDIX - MOTOR PARAMETERS}

\begin{tabular}{|c|c|}
\hline Parameter & Value \\
\hline$L$ & $0.000102 \mathrm{H}$ \\
\hline$R$ & $0.022 \Omega$ \\
\hline$k_{e}$ & $0.23 \mathrm{~V} / \mathrm{rad} / \mathrm{s}$ \\
\hline$V_{\max }$ & $87 \mathrm{~V}$ \\
\hline$I_{\max }$ & $200 \mathrm{~A}(\mathrm{rms})$ \\
\hline$T_{\max }$ & $87 \mathrm{Nm}$ \\
\hline
\end{tabular}

\section{REFERENCES}

1. Verl, A., and M. Bodson, "Torque Maximisation for Permanent Magnet Synchronous Motors," IEEE Trans. Contr. Syst. Technol., Vol. 6, No. 6, pp. 740744 (1998).

2. Shi, J. and Y.-S. Lu, "Field-weakening Operation of Cylindrical Permanent Magnet Motors," Proc. IEEE Conf. Contr. Appl., Dearborn, MI, pp. 864-869 (1996).

3. Bodson, M., J. Chiasson, and R.T. Novotnak, "A Systematic Approach to Selecting Optimal Flux References in Induction Motors," IEEE Trans. Contr. Syst. Technol., Vol. 3, No. 4, pp. 388-397 (1995).

4. Miller, T.J.E., "Brushless Permanent Magnet and Reluctance Motor Drives," University Press, Oxford (1993).

5. Pillay, P. and R. Krishnan, "Modelling, Simulation, and Analysis of Permanent Magnet Motor Drives,
Part 1: The Permanent Magnet Synchronous Motor Drive," IEEE Trans. Ind. Appl., Vol. 25, No. 2, pp. 265-273 (1989).

6. Hindmarsh, J., Electrical Machines and Drives, Pergamon Press Ltd., Oxford, U.K. (1985).

7. Kim, J.M. and S.K. Sul, "Speed Control of Interior Permanent Magnet Synchronous Motor Drive for the Flux-Weakening Operation," IEEE Trans. Ind. Appl., Vol. 33, No. 1, pp. 43-48 (1997).

8. Stewart, P. and V. Kadirkamanathan, "Dynamic Control of Permanent Magnet Synchronous Motors in Automotive Drive Applications," Proc. IEEE Ame. Contr. Conf., San Diego, USA, pp. 1677-1681 (1999).

9. Blauch, A., M. Bodson, and J. Chiasson, "Highspeed Parameter Estimation of Stepper Motors," IEEE Trans. Contr. Syst. Technol., Vol. 1, pp. 270-279 (1993).

10. Bose, B.K., Ed., Power Electronics and Variable Speed Drives, IEEE Press, Piscataway NJ., (chap 6) (1996).

11. Leonhard, W., Control of Electrical Drives, Springer Verlag, Berlin, $2^{\text {rd }}$ Ed. (1996).

12. Boldea, S.A., Electrical Drives, CRC Press, Boca Raton (1998).

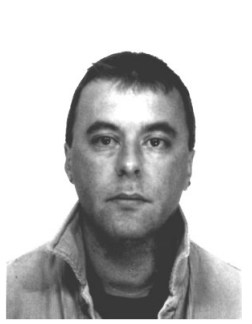

Dr. Paul Stewart gained the Beng in Electronic Control and Systems Engineering in 1996, and the $\mathrm{PhD}$ on Permanent Magnet AC Drives in 1999, both from the University of Sheffield in the United Kingdom.

Dr. Stewart is currently researching the application of electronically actuated inlet and exhaust valves for internal combustion engines, applying multi objective evolutionary algorithms to the problem of optimal electromagnetic design. 